\title{
Reconfiguration Criterion for Fault-Tolerant Control
}

\author{
Inseok Yang, ${ }^{1}$ Dongik Lee, ${ }^{2}$ and Dong Seog Han ${ }^{2}$ \\ ${ }^{1}$ Center for ICT \& Automobile Convergence, Kyungpook National University, Daegu 702-701, Republic of Korea \\ ${ }^{2}$ School of Electronics Engineering, Kyungpook National University, Daegu 702-701, Republic of Korea \\ Correspondence should be addressed to Dong Seog Han; dshan@knu.ac.kr
}

Received 31 July 2014; Accepted 19 December 2014

Academic Editor: Qingling Zhang

Copyright (C) 2015 Inseok Yang et al. This is an open access article distributed under the Creative Commons Attribution License, which permits unrestricted use, distribution, and reproduction in any medium, provided the original work is properly cited.

The reconfiguration criterion for tolerating actuator fault is proposed. The proposed reconfiguration criterion analyzes the relationship between normal actuators and the system states that are directly affected by faulty actuators. So the proposed criterion provides the possibilities of fault-tolerance. Moreover, it also provides the required number of redundant normal actuators.

\section{Introduction}

Faults occurring in systems such as automotive vehicles and aircrafts cause a catastrophic accident that leads to loss of property, life, and so forth. To avoid an accident caused by faults, many systems require a high level of dependability. Adopting redundant actuators has been considered as an efficient method of achieving the required dependability. Conventionally, redundant actuators are considered as backup systems if the primary ones operate normally. But if faults occur in the primary actuators, then redundant ones are activated as main actuators. However, adopting redundant actuators leads to the losses of fuel, space, cost, and weight during normal operation.

In contrast to adopting hardware redundancies such as secondary actuators, software based fault accommodation methods have been proposed for the last 30 years. The goal of these methods is to provide the feasible control input in order to maintain the normal performance. For this reason, these methods are defined as fault-tolerant control (FTC) or reconfiguration. There have been proposed various fault-tolerant control techniques: pseudoinverse [1], model reference adaptive control [2], sliding mode control [3-5], multiple model switching and tuning [6], control allocation $[3,7-10]$, and so forth.

However, most of the proposed methods shown above consider the reconfiguration ability. This means that although the proposed reconfiguration methods can accommodate faults theoretically and practically, there are some faulty systems that cannot be tolerated. Generally, reconfiguration possibility highly depends on the relationship between the faulty states and controllable normal inputs. If a faulty system cannot take sufficient controllable inputs related to the faulty states, then the goal of reconfiguration must be changed to achieve stabilization of the faulty system in order to avoid structural damage. This paper proposes the reconfiguration condition that provides the possibility of fault-tolerance. By explicitly analyzing the relationship between the faulty states and normal inputs, the condition also proposes the required number of redundant actuators that can achieve fault-tolerance.

\section{General Dynamic Model of Actuator Faulty System}

The response of a faulty actuator can be categorized into one of four types: Lock-in-Place (LiP), Hardover, Float, and Loss of Effectiveness (LoE) [11]. Figure 1 shows the typical examples of these fault types. In this figure, faults such as LiP, Hardover, and Float lead an actuator to stopping at one position or diverging to upper-/lower-saturation position without any consideration of the input commands. So these faults are defined as total faults. In contrast to total faults, the response of LoE fault degrades the performance relative to 


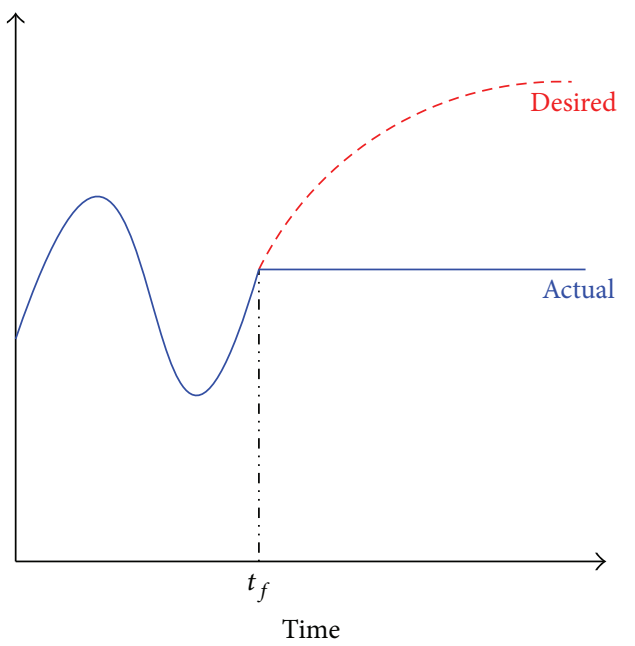

(a) Lock-in-Place

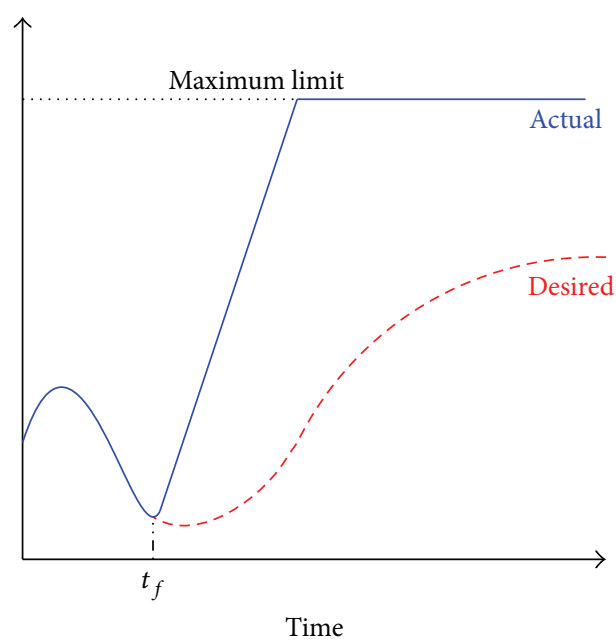

(c) Hardover

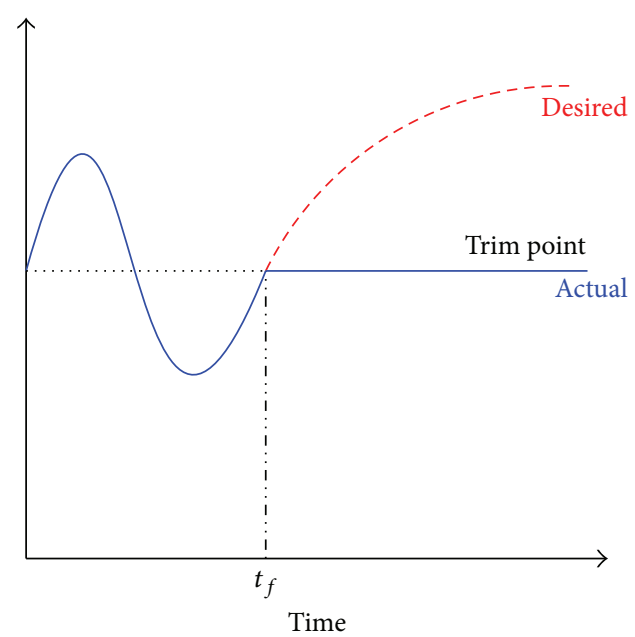

(b) Float

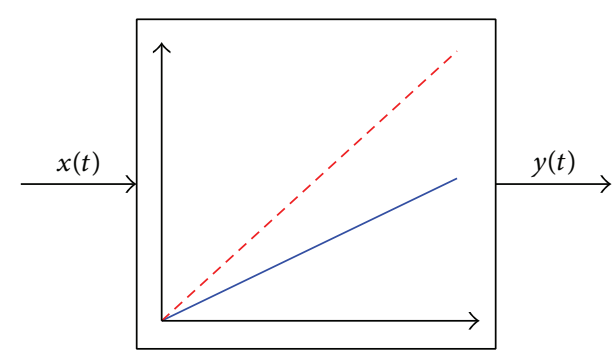

(d) Loss of Effectiveness

FIgURE 1: Typical failures of actuator [11].

its desired (normal) output. Hence, the general response of a faulty actuator can be represented as follows:

$$
u_{i}=\underbrace{\llbracket \gamma_{i} \rrbracket \gamma_{i} u_{i}^{\text {NORMAL }}}_{\text {LoE fault term }}+\underbrace{\left(1-\llbracket \gamma_{i} \rrbracket\right) u_{i}^{\text {TOTAL }}}_{\text {Total fault term }},
$$

where the subscript $i$ indicates the $i$ th actuator and $\llbracket$. \ is the smallest integer greater than or equal to $\cdot$. And $\gamma_{i}$ is the performance degradation factor represented by a quantitative value in $[0,1]$ according to the degraded performance:

$$
\gamma_{i}= \begin{cases}0, & \text { if total fault occurs } \\ \varepsilon(0<\varepsilon<1), & \text { if LoE fault occurs } \\ 1, & \text { if no fault occurs. }\end{cases}
$$

And $u_{i}^{\text {NORMAL }}$ denotes the desired normal position shown as a dotted red line in Figure $1(\mathrm{~d})$, and $u_{i}^{\text {TOTAL }}$ denotes the faulty position depicted as a blue line in Figures 1(a), 1(b), and 1(c).
For example, if the $i$ th actuator is operated normally, then $\gamma_{i}=$ 1 , so $\delta_{i}=u_{i}^{\text {NORMAL }}$. And if $50 \%$ of LoE failure occurs on the $i$ th actuator, then $\gamma_{i}=0.5$, so $u_{i}=0.5 u_{i}^{\text {NORMAL }}$.

For the total number of actuators $m$, suppose faults are occurring on $k(1 \leq k \leq m)$ actuators; then, the general dynamics of faulty actuators yields

$$
\begin{aligned}
{\left[\begin{array}{c}
u_{1} \\
u_{2} \\
\vdots \\
u_{k}
\end{array}\right]=} & {\left[\begin{array}{cccc}
\llbracket \gamma_{1} \rrbracket \gamma_{1} & 0 & \cdots & 0 \\
0 & \llbracket \gamma_{2} \rrbracket \gamma_{2} & \cdots & 0 \\
\vdots & \vdots & \ddots & \vdots \\
0 & 0 & \cdots & \llbracket \gamma_{k} \rrbracket \gamma_{k}
\end{array}\right]\left[\begin{array}{c}
u_{1}^{\text {NORMAL }} \\
u_{2}^{\text {NORMAL }} \\
\vdots \\
u_{k}^{\text {NORMAL }}
\end{array}\right] } \\
+ & {\left[\begin{array}{cccc}
1-\llbracket \gamma_{1} \rrbracket & 0 & \cdots & 0 \\
0 & 1-\llbracket \gamma_{2} \rrbracket & \cdots & 0 \\
\vdots & \vdots & \ddots & \vdots \\
0 & 0 & \cdots & 1-\llbracket \gamma_{k} \rrbracket
\end{array}\right]\left[\begin{array}{c}
u_{1}^{\text {TOTAL }} \\
u_{2}^{\text {TOTAL }} \\
\vdots \\
u_{k}^{\text {TOTAL }}
\end{array}\right] }
\end{aligned}
$$




$$
\begin{aligned}
& =\left[\begin{array}{cccc}
\llbracket \gamma_{1} \rrbracket & 0 & \cdots & 0 \\
0 & \llbracket \gamma_{2} \rrbracket & \cdots & 0 \\
\vdots & \vdots & \ddots & \vdots \\
0 & 0 & \cdots & \llbracket \gamma_{k} \rrbracket
\end{array}\right]\left[\begin{array}{cccc}
\gamma_{1} & 0 & \cdots & 0 \\
0 & \gamma_{2} & \cdots & 0 \\
\vdots & \vdots & \ddots & \vdots \\
0 & 0 & \cdots & \gamma_{k}
\end{array}\right] \\
& \times\left[\begin{array}{c}
u_{1}^{\text {NORMAL }} \\
u_{2}^{\text {NORMAL }} \\
\vdots \\
u_{k}^{\text {NORMAL }}
\end{array}\right] \\
& +\left\{\left[\begin{array}{cccc}
1 & 0 & \cdots & 0 \\
0 & 1 & \cdots & 0 \\
\vdots & \vdots & \ddots & \vdots \\
0 & 0 & \cdots & 1
\end{array}\right]-\left[\begin{array}{cccc}
\llbracket \gamma_{1} \rrbracket & 0 & \cdots & 0 \\
0 & \llbracket \gamma_{2} \rrbracket & \cdots & 0 \\
\vdots & \vdots & \ddots & \vdots \\
0 & 0 & \cdots & \llbracket \gamma_{k} \rrbracket
\end{array}\right]\right\} \\
& \times\left[\begin{array}{c}
u_{1}^{\text {TOTAL }} \\
u_{2}^{\text {TOTAL }} \\
\vdots \\
u_{k}^{\text {TOTAL }}
\end{array}\right] \\
& \underset{\text { Let }}{\Longrightarrow} \mathbf{u}_{1}^{F}=\llbracket \boldsymbol{\Gamma}^{F} \rrbracket \boldsymbol{\Gamma}^{F} \mathbf{u}^{F, \text { NORMAL }}+\left(\mathbf{I}_{k}-\llbracket \boldsymbol{\Gamma}^{F} \rrbracket\right) \mathbf{u}^{F, \text { TOTAL }},
\end{aligned}
$$

where $\mathbf{u}^{\text {F,NORMAL }}=\left[u_{1}^{\text {NORMAL }}, u_{2}^{\text {NORMAL }}, \ldots, u_{k}^{\text {NORMAL }}\right]^{T}$ and $\mathbf{u}^{F, \text { TOTAL }}=\left[u_{1}^{\text {TOTAL }}, u_{2}^{\text {TOTAL }}, \ldots, u_{k}^{\text {TOTAL }}\right]^{T}$. And $\mathbf{I}_{k}$ is a $(k \times k)$ identity matrix. Moreover,

$$
\begin{gathered}
\boldsymbol{\Gamma}^{F}=\left[\begin{array}{cccc}
\gamma_{1} & 0 & \cdots & 0 \\
0 & \gamma_{2} & \cdots & 0 \\
\vdots & \vdots & \ddots & \vdots \\
0 & 0 & \cdots & \gamma_{k}
\end{array}\right], \\
\llbracket \Gamma^{F} \rrbracket=\left[\begin{array}{cccc}
\llbracket \gamma_{1} \rrbracket & 0 & \cdots & 0 \\
0 & \llbracket \gamma_{2} \rrbracket & \cdots & 0 \\
\vdots & \vdots & \ddots & \vdots \\
0 & 0 & \cdots & \llbracket \gamma_{k} \rrbracket
\end{array}\right] .
\end{gathered}
$$

From (3), the general responses of actuators including faulty and normal actuators can be represented as follows:

$$
\begin{aligned}
{\left[\begin{array}{c}
\mathbf{u}_{1}^{F} \\
\mathbf{u}_{2}^{N}
\end{array}\right]=} & {\left[\begin{array}{cc}
\llbracket \Gamma^{F} \rrbracket & \mathbf{0}_{k \times(m-k)} \\
\mathbf{0}_{(m-k) \times k} & \llbracket \mathbf{I}_{m-k} \rrbracket
\end{array}\right]\left[\begin{array}{cc}
\Gamma^{F} & \mathbf{0}_{k \times(m-k)} \\
\mathbf{0}_{(m-k) \times k} & \mathbf{I}_{m-k}
\end{array}\right] } \\
& \times\left[\begin{array}{c}
\mathbf{u}^{F, N O R M A L} \\
\mathbf{u}^{N, \text {NORMAL }}
\end{array}\right]
\end{aligned}
$$

$$
\begin{aligned}
& +\left\{\left[\begin{array}{cc}
\mathbf{I}_{k} & \mathbf{0}_{k \times(m-k)} \\
\mathbf{0}_{(m-k) \times k} & \mathbf{I}_{m-k}
\end{array}\right]-\left[\begin{array}{cc}
\llbracket \Gamma^{F} \rrbracket & \mathbf{0}_{k \times(m-k)} \\
\mathbf{0}_{(m-k) \times k} & \llbracket \mathbf{I}_{m-k} \rrbracket
\end{array}\right]\right\} \\
& \times\left[\begin{array}{c}
\mathbf{u}^{F, \mathrm{TOTAL}} \\
\mathbf{0}_{(m-k) \times 1}
\end{array}\right] \\
& \underset{\text { Let }}{\Longrightarrow} \mathbf{u}=\llbracket \boldsymbol{\Gamma} \rrbracket \boldsymbol{\Gamma} \mathbf{u}^{\text {NORMAL }}+\left(\mathbf{I}_{m}-\llbracket \boldsymbol{\Gamma} \rrbracket\right) \mathbf{u}^{\mathrm{TOTAL}},
\end{aligned}
$$

where $\mathbf{u}_{2}^{N} \in \mathbf{R}^{m-k}$ is a set of normal actuators $\mathbf{u}^{N, N O R M A L}$ and $\mathbf{I}_{m}$ is an $(m \times m)$-identity matrix. And

$$
\begin{gathered}
\mathbf{u}=\left[\begin{array}{c}
\mathbf{u}_{1}^{F} \\
\mathbf{u}_{2}^{N}
\end{array}\right], \quad \mathbf{u}^{\text {NORMAL }}=\left[\begin{array}{c}
\mathbf{u}^{F, N O R M A L} \\
\mathbf{u}^{N, \text {NORMAL}}
\end{array}\right], \\
\mathbf{u}^{\mathrm{TOTAL}}=\left[\begin{array}{c}
\mathbf{u}^{F, \mathrm{TOTAL}} \\
\mathbf{0}_{(m-k) \times 1}
\end{array}\right], \quad \boldsymbol{\Gamma}=\left[\begin{array}{cc}
\boldsymbol{\Gamma}^{F} & \mathbf{0}_{k \times(m-k)} \\
\mathbf{0}_{(m-k) \times k} & \mathbf{I}_{m-k}
\end{array}\right], \\
\llbracket \boldsymbol{\Gamma} \rrbracket=\left[\begin{array}{cc}
\llbracket \boldsymbol{\Gamma}^{F} \rrbracket & \llbracket \mathbf{0}_{k \times(m-k)} \rrbracket \\
\llbracket \mathbf{0}_{(m-k) \times k} \rrbracket & \llbracket \mathbf{I}_{m-k} \rrbracket
\end{array}\right]=\left[\begin{array}{cc}
\llbracket \boldsymbol{\Gamma}^{F} \rrbracket & \mathbf{0}_{k \times(m-k)} \\
\mathbf{0}_{(m-k) \times k} & \mathbf{I}_{m-k}
\end{array}\right] .
\end{gathered}
$$

If no faults are occurring in actuators, then $\boldsymbol{\Gamma}^{F}=\mathbf{I}_{k}$ and $\llbracket \boldsymbol{\Gamma}^{F} \rrbracket=\mathbf{I}_{k}$ in (5) and $\boldsymbol{\Gamma}=\mathbf{I}_{m}$ and $\llbracket \boldsymbol{\Gamma} \rrbracket=\mathbf{I}_{m}$ in (6). Hence, $\mathbf{u}=\mathbf{u}^{\text {NORMAL }}$

To analyze the general dynamics of a faulty system, let us consider the following linearized model:

$$
\dot{\mathbf{x}}=\mathbf{A x}+\mathbf{B u},
$$

where $\mathbf{x} \in \mathbf{R}^{n}$ is a state vector and $\mathbf{u} \in \mathbf{R}^{m}$ is an input vector. Then the generalized model including $k$-faulty actuators yields

$$
\begin{aligned}
\dot{\mathbf{x}}= & \mathbf{A} \mathbf{x}+\mathbf{B u} \\
= & \mathbf{A} \mathbf{x}+\mathbf{B}\left[\llbracket \boldsymbol{\Gamma} \rrbracket \boldsymbol{\Gamma} \mathbf{u}^{\text {NORMAL }}+\left(\mathbf{I}_{m}-\llbracket \boldsymbol{\Gamma} \rrbracket\right) \mathbf{u}^{\text {TOTAL }}\right] \\
= & \mathbf{A} \mathbf{x}+\left[\begin{array}{ll}
\mathbf{B}_{1}^{F} & \mathbf{B}_{2}^{N}
\end{array}\right] \\
& \times\left\{\left[\begin{array}{ll}
\llbracket \boldsymbol{\Gamma}^{F} \rrbracket \boldsymbol{\Gamma}^{F} & \mathbf{0}_{k \times(m-k)} \\
\mathbf{0}_{(m-k) \times k} & \mathbf{I}_{m-k}
\end{array}\right]\left[\begin{array}{l}
\mathbf{u}^{F, \mathrm{NORMAL}} \\
\mathbf{u}^{N, \mathrm{NORMAL}}
\end{array}\right]\right. \\
& \left.+\left[\begin{array}{cc}
\left(\mathbf{I}_{k}-\llbracket \boldsymbol{\Gamma}^{F} \rrbracket\right) & \mathbf{0}_{k \times(m-k)} \\
\mathbf{0}_{(m-k) \times k} & \mathbf{0}_{(m-k) \times(m-k)}
\end{array}\right]\left[\begin{array}{l}
\mathbf{u}^{F, \mathrm{TOTAL}} \\
\mathbf{0}_{(m-k) \times 1}
\end{array}\right]\right\} \\
= & \mathbf{A x}+\mathbf{B}_{2}^{N} \mathbf{u}^{N, \mathrm{NORMAL}} \\
+ & \mathbf{B}_{1}^{F}\left[\llbracket \boldsymbol{\Gamma}^{F} \rrbracket \boldsymbol{\Gamma}^{F} \mathbf{u}^{F, \mathrm{NORMAL}}+\left(\mathbf{I}_{k}-\llbracket \boldsymbol{\Gamma}^{F} \rrbracket\right) \mathbf{u}^{F, \mathrm{TOTAL}}\right],
\end{aligned}
$$

where $\mathbf{B}_{1}^{F} \in \mathbf{R}^{n \times k}$ and $\mathbf{B}_{2}^{N} \in \mathbf{R}^{n \times(m-k)}$ are fault and normal input distribution matrices, respectively; that is, for the input distribution matrix denoted by $\mathbf{B}=\left[\mathbf{b}_{1}, \mathbf{b}_{2}, \ldots, \mathbf{b}_{m}\right]$, if failures are occurring on both $i$ th and $j$ th $(i \leq j)$ actuators, then $\mathbf{B}_{1}^{F}=\left[\mathbf{b}_{i}, \mathbf{b}_{j}\right]$ and $\mathbf{B}_{2}^{N}=\left[\mathbf{b}_{1}, \mathbf{b}_{2}, \ldots, \mathbf{b}_{i-1}, \mathbf{b}_{i+1}, \ldots, \mathbf{b}_{j-1}, \mathbf{b}_{j+1}\right.$, $\left.\ldots, \mathbf{b}_{m}\right]$. 


\section{The Proposed Fault-Tolerant Control Allocation Method}

In this section, the reconfiguration criterion is proposed. The proposed criterion determines whether the faulty system has sufficient normal actuators enough to accommodate faults occurring on several actuators. To analyze the criterion, the effects of faulty actuators are analyzed first. Next, the reconfiguration criterion is proposed. And then the minimum number of redundant actuators for compensating the effects of faulty actuators is proposed in the last of this section.

3.1. Effects of Faulty Actuators. In the generalized faulty system model provided in the previous section, faults on actuators directly affect the actuator term. To analyze the effects of actuator faults, the virtual input vector $\mathbf{v} \in \mathbf{R}^{n}$ is employed:

$$
\mathbf{v} \underset{\text { Let }}{=}\left[\begin{array}{ll}
\mathbf{B}_{1}^{F} & \mathbf{B}_{2}^{N}
\end{array}\right]\left[\begin{array}{c}
\mathbf{u}_{1}^{F} \\
\mathbf{u}_{2}^{N}
\end{array}\right] .
$$

In (9), not all elements of $\mathbf{v}$ are affected by faulty actuators. To classify $\mathbf{v}$ into the fault-affected term $\mathbf{v}_{1}^{F}$ and the faultfree (normal) term $\mathbf{v}_{2}^{N}$, rearrange the row vectors of the input distribution matrix as

$$
\left[\begin{array}{c}
\mathbf{v}_{1}^{F} \\
\mathbf{v}_{2}^{N}
\end{array}\right]=\left[\begin{array}{cc}
\mathbf{B}_{11}^{F} & \mathbf{B}_{12}^{N} \\
\mathbf{0} & \mathbf{B}_{22}^{N}
\end{array}\right]\left[\begin{array}{c}
\mathbf{u}_{1}^{F} \\
\mathbf{u}_{2}^{N}
\end{array}\right],
$$

where $\mathbf{v}_{1}^{F} \in \mathbf{R}^{r}$ and $\mathbf{v}_{2}^{N} \in \mathbf{R}^{(n-r)}$; that is, the number system states affected by faulty actuators are assumed to be $r$. And $\mathbf{B}_{11}^{F} \in \mathbf{R}^{r \times k}, \mathbf{B}_{12}^{N} \in \mathbf{R}^{r \times(m-k)}$, and $\mathbf{B}_{22}^{N} \in \mathbf{R}^{(n-r) \times(m-k)}$. From (3) and (10), the virtual input can also be represented by the combination of fault types as follows:

$$
\begin{aligned}
\mathbf{v}_{1}^{F}= & \mathbf{B}_{11}^{F}\left[\llbracket \boldsymbol{\Gamma}^{F} \rrbracket \boldsymbol{\Gamma}^{F} \mathbf{u}^{F, \mathrm{LOE}}+\left(\mathbf{I}_{k}-\llbracket \boldsymbol{\Gamma}^{F} \rrbracket\right) \mathbf{u}^{F, \mathrm{TOTAL}}\right] \\
& +\mathbf{B}_{12}^{N} \mathbf{u}^{N, \mathrm{LOE}}, \\
\mathbf{v}_{2}^{N}= & \mathbf{B}_{22}^{N} \mathbf{u}^{N, \mathrm{LOE}} .
\end{aligned}
$$

Let us denote the input error vector by $\mathbf{e}^{u}=\mathbf{u}_{1}^{F}-\mathbf{u}_{1}^{N}$ for the expected normal input vector $\mathbf{u}_{1}^{N}$ represented as a dotted red line in Figure 1(d). And denote the corresponding virtual input error vector by $\mathbf{e}^{v}=\mathbf{v}_{1}^{F}-\mathbf{v}_{1}^{N}$ for the expected normal virtual vector $\mathbf{v}_{1}^{N}=\mathbf{B}_{11}^{F} \mathbf{u}_{1}^{N}+\mathbf{B}_{12}^{N} \mathbf{u}_{2}^{N}$. Then the effects of faulty actuators can be represented as follows:

$$
\begin{aligned}
{\left[\begin{array}{c}
\mathbf{v}_{1}^{F} \\
\mathbf{v}_{2}^{N}
\end{array}\right]-\left[\begin{array}{c}
\mathbf{v}_{1}^{N} \\
\mathbf{v}_{2}^{N}
\end{array}\right] } & =\left[\begin{array}{cc}
\mathbf{B}_{11}^{F} & \mathbf{B}_{12}^{N} \\
\mathbf{0}_{(n-r) \times k} & \mathbf{B}_{22}^{N}
\end{array}\right]\left\{\left[\begin{array}{c}
\mathbf{u}_{1}^{F} \\
\mathbf{u}_{2}^{N}
\end{array}\right]-\left[\begin{array}{c}
\mathbf{u}_{1}^{N} \\
\mathbf{u}_{2}^{N}
\end{array}\right]\right\} \\
& \Longrightarrow\left[\begin{array}{c}
\mathbf{e}^{v} \\
\mathbf{0}_{(n-r)}
\end{array}\right]=\left[\begin{array}{cc}
\mathbf{B}_{11}^{F} & \mathbf{B}_{12}^{N} \\
\mathbf{0}_{(n-r) \times k} & \mathbf{B}_{22}^{N}
\end{array}\right]\left[\begin{array}{c}
\mathbf{e}^{u} \\
\mathbf{0}_{(m-k)}
\end{array}\right] .
\end{aligned}
$$

Due to faults on $k$-actuators, $\mathbf{e}^{v} \in \mathbf{R}^{r}$ cannot be zero. By (3), the virtual input error vector represented by the combination of fault types yields

$$
\begin{aligned}
\mathbf{e}^{v} & =\mathbf{B}_{11}^{F} \mathbf{e}^{u}=\mathbf{B}_{11}^{F}\left(\mathbf{u}_{1}^{F}-\mathbf{u}_{1}^{N}\right) \\
& =\mathbf{B}_{11}^{F}\left\{\llbracket \Gamma^{F} \rrbracket \boldsymbol{\Gamma}^{F} \mathbf{u}^{F, \mathrm{LOE}}+\left(\mathbf{I}_{k}-\llbracket \boldsymbol{\Gamma}^{F} \rrbracket\right) \mathbf{u}^{F, \mathrm{TOTAL}}-\mathbf{u}_{1}^{N}\right\} .
\end{aligned}
$$

3.2. Reconfiguration Criterion. In this subsection, the faulttolerant control problem is introduced, and then the reconfiguration criterion is proposed. In (12), for the faulty-actuated system, the only controllable actuators are $\mathbf{u}_{2}^{N}$. This leads to the fact that the relationship between $\mathbf{u}_{1}^{F}$ and $\mathbf{u}_{2}^{N}$ can be efficiently used to compensate the effects of faulty actuators. Hence, the fault-tolerant control problem can be stated as finding the control input $\mathbf{u}_{2}^{N}$ that can reduce $\mathbf{e}^{v}$. Let us denote the fault-tolerant control input by $\mathbf{u}^{R} \in \mathbf{R}^{m-k}$. Without loss of generality, it is assumed that $\mathbf{u}^{R}=\mathbf{u}_{2}^{N}+\mathbf{u}_{2}^{R}$ to simplify the problem. Then the input-virtual input relationship controlled by $\mathbf{u}^{R}$ can be represented as follows:

$$
\begin{aligned}
{\left[\begin{array}{c}
\mathbf{e}^{v} \\
\mathbf{0}_{(n-r)}
\end{array}\right] } & =\left[\begin{array}{cc}
\mathbf{B}_{11}^{F} & \mathbf{B}_{12}^{N} \\
\mathbf{0} & \mathbf{B}_{22}^{N}
\end{array}\right]\left\{\left[\begin{array}{l}
\mathbf{u}_{1}^{F} \\
\mathbf{u}^{R}
\end{array}\right]-\left[\begin{array}{c}
\mathbf{u}_{1}^{N} \\
\mathbf{u}_{2}^{N}
\end{array}\right]\right\} \\
& \underset{\mathbf{e}^{v} \rightarrow \mathbf{0}_{r}}{\Longrightarrow}\left[\begin{array}{c}
\mathbf{0}_{r} \\
\mathbf{0}_{(n-r)}
\end{array}\right]=\left[\begin{array}{cc}
\mathbf{B}_{11}^{F} & \mathbf{B}_{12}^{N} \\
\mathbf{0} & \mathbf{B}_{22}^{N}
\end{array}\right]\left\{\left[\begin{array}{c}
\mathbf{u}_{1}^{F} \\
\mathbf{u}_{2}^{N}+\mathbf{u}_{2}^{R}
\end{array}\right]-\left[\begin{array}{c}
\mathbf{u}_{1}^{N} \\
\mathbf{u}_{2}^{N}
\end{array}\right]\right\} \\
& \Longrightarrow\left[\begin{array}{c}
\mathbf{0}_{r} \\
\mathbf{0}_{(n-r)}
\end{array}\right]=\left[\begin{array}{cc}
\mathbf{B}_{11}^{F} & \mathbf{B}_{12}^{N} \\
\mathbf{0}_{(n-r) \times k} & \mathbf{B}_{22}^{N}
\end{array}\right]\left[\begin{array}{c}
\mathbf{e}^{u} \\
\mathbf{u}_{2}^{R}
\end{array}\right] .
\end{aligned}
$$

It is worth noting that (14) shows the conceptual description of fault-tolerant control. The possibility of tolerating the effects of faulty actuators highly depends on the relationship between $\mathbf{u}_{1}^{F}$ and $\mathbf{u}_{2}^{N}$. This means that the characteristics of matrices $\mathbf{B}_{11}^{F}$ and $\mathbf{B}_{12}^{N}$ determine the possibility of faulttolerance. In this paper, the conditions for tolerating the effects of faults based on the characteristics of matrices $\mathbf{B}_{11}^{F}$ and $\mathbf{B}_{12}^{N}$ are proposed as the reconfiguration criterion. Hence, the proposed reconfiguration criterion describes the relationship between normal actuators and effects of faulty actuators. Moreover, this reconfiguration criterion determines whether there are a sufficient number of normal actuators that can accommodate faults occurring on actuators.

Before proposing the reconfiguration criterion, rearrange the input distribution matrix $\left[\begin{array}{ll}\mathbf{B}_{1}^{F} & \mathbf{B}_{2}^{N}\end{array}\right]$ as follows: for $n_{p} \leq n$ and $m_{q}=m-k$, 


$$
\begin{aligned}
& \mathbf{B}_{1}^{F}=\left[\begin{array}{cccc}
b_{11}^{F} & b_{12}^{F} & \cdots & b_{1 k}^{F} \\
b_{21}^{F} & b_{22}^{F} & \cdots & b_{2 k}^{F} \\
\vdots & \vdots & \ddots & \vdots \\
b_{r 1}^{F} & b_{r 2}^{F} & \cdots & b_{r k}^{F} \\
0 & 0 & \cdots & 0 \\
\vdots & \vdots & \ddots & \vdots \\
0 & 0 & \cdots & 0
\end{array}\right], \\
& \mathbf{B}_{2}^{N}=\left[\begin{array}{cccccccccc}
b_{11}^{N} & b_{12}^{N} & \cdots & b_{1 m_{1}}^{N} & b_{1\left(m_{1}+1\right)}^{N} & \cdots & b_{1 m_{2}}^{N} & b_{1\left(m_{2}+1\right)}^{N} & \cdots & b_{1 m_{q}}^{N} \\
b_{21}^{N} & b_{22}^{N} & \cdots & b_{2 m_{1}}^{N} & b_{2\left(m_{1}+1\right)}^{N} & \cdots & b_{2 m_{2}}^{N} & b_{1\left(m_{2}+1\right)}^{N} & \cdots & b_{2 m_{q}}^{N} \\
\vdots & \vdots & \ddots & \vdots & \vdots & \ddots & \vdots & \vdots & \ddots & \vdots \\
b_{n_{1} 1}^{N} & b_{n_{1} 2}^{N} & \cdots & b_{n_{1} m_{1}}^{N} & b_{n_{1}\left(m_{1}+1\right)}^{N} & \cdots & b_{n_{1} m_{2}}^{N} & b_{n_{1}\left(m_{2}+1\right)}^{N} & \cdots & b_{n_{1} m_{q}}^{N} \\
0 & 0 & \cdots & 0 & b_{\left(n_{1}+1\right)\left(m_{1}+1\right)}^{N} & \cdots & b_{\left(n_{1}+1\right) m_{2}}^{N} & b_{\left(n_{1}+1\right)\left(m_{2}+1\right)}^{N} & \cdots & b_{\left(n_{1}+1\right) m_{q}}^{N} \\
\vdots & \vdots & \ddots & \vdots & \vdots & \ddots & \vdots & \vdots & \ddots & \vdots \\
0 & 0 & \cdots & 0 & b_{n_{2}\left(m_{1}+1\right)}^{N} & \cdots & b_{n_{2} m_{2}}^{N} & b_{n_{2}\left(m_{2}+1\right)}^{N} & \cdots & b_{n_{2} m_{q}}^{N} \\
0 & 0 & \cdots & 0 & 0 & \cdots & 0 & b_{\left(n_{2}+1\right)\left(m_{2}+1\right)}^{N} & \cdots & b_{\left(n_{2}+1\right) m_{q}}^{N} \\
\vdots & \vdots & \ddots & \vdots & \vdots & \ddots & \vdots & \vdots & \ddots & \vdots \\
0 & 0 & \cdots & 0 & 0 & \cdots & 0 & 0 & \cdots & b_{n_{p} m_{q}}^{N} \\
0 & 0 & \cdots & 0 & 0 & \cdots & 0 & 0 & \cdots & 0 \\
\vdots & \vdots & \ddots & \vdots & \vdots & \ddots & \vdots & \vdots & \ddots & \vdots \\
0 & 0 & \cdots & 0 & 0 & \cdots & 0 & 0 & \cdots & 0
\end{array}\right] \\
& =\left[\begin{array}{cccccccc}
\widetilde{\mathbf{B}}_{11}^{N} & \widetilde{\mathbf{B}}_{12}^{N} & \widetilde{\mathbf{B}}_{13}^{N} & \cdots & \widetilde{\mathbf{B}}_{1 l}^{N} & \widetilde{\mathbf{B}}_{1(l+1)}^{N} & \cdots & \widetilde{\mathbf{B}}_{1 q}^{N} \\
\mathbf{0} & \widetilde{\mathbf{B}}_{22}^{N} & \widetilde{\mathbf{B}}_{23}^{N} & \cdots & \widetilde{\mathbf{B}}_{2 l}^{N} & \widetilde{\mathbf{B}}_{2(l+1)}^{N} & \cdots & \widetilde{\mathbf{B}}_{2 q}^{N} \\
\mathbf{0} & \mathbf{0} & \widetilde{\mathbf{B}}_{33}^{N} & \cdots & \widetilde{\mathbf{B}}_{3 l}^{N} & \widetilde{\mathbf{B}}_{3(l+1)}^{N} & \cdots & \widetilde{\mathbf{B}}_{3 q}^{N} \\
\vdots & \vdots & \vdots & \ddots & \vdots & \vdots & \ddots & \vdots \\
\mathbf{0} & \mathbf{0} & \mathbf{0} & \cdots & \widetilde{\mathbf{B}}_{l l}^{N} & \widetilde{\mathbf{B}}_{l(l+1)}^{N} & \cdots & \widetilde{\mathbf{B}}_{l q}^{N} \\
\mathbf{0} & \mathbf{0} & \mathbf{0} & \cdots & \mathbf{0} & \widetilde{\mathbf{B}}_{(l+1)(l+1)}^{N} & \cdots & \widetilde{\mathbf{B}}_{(l+1) q}^{N} \\
\vdots & \vdots & \vdots & \ddots & \vdots & \vdots & \ddots & \vdots \\
\mathbf{0} & \mathbf{0} & \mathbf{0} & \cdots & \mathbf{0} & \mathbf{0} & \cdots & \widetilde{\mathbf{B}}_{p q}^{N} \\
\mathbf{0} & \mathbf{0} & \mathbf{0} & \cdots & \mathbf{0} & \mathbf{0} & \cdots & \mathbf{0}
\end{array}\right] .
\end{aligned}
$$

For $1 \leq i \leq p$ and $1 \leq j \leq q$, assume that $n_{0}=m_{0}=0$. And set $\widetilde{\mathbf{B}}_{i j}^{N}$ as

$$
\begin{aligned}
\widetilde{\mathbf{B}}_{i j}^{N} & =\left[\begin{array}{cccc}
b_{\left(n_{i-1}+1\right)\left(m_{j-1}+1\right)}^{N} & b_{\left(n_{i-1}+1\right)\left(m_{j-1}+2\right)}^{N} & \cdots & b_{\left(n_{i-1}+1\right) m_{j}}^{N} \\
b_{\left(n_{i-1}+2\right)\left(m_{j-1}+1\right)}^{N} & b_{\left(n_{i-1}+2\right)\left(m_{j-1}+2\right)}^{N} & \cdots & b_{\left(n_{i-1}+2\right) m_{j}}^{N} \\
\vdots & \vdots & \ddots & \vdots \\
b_{n_{i}\left(m_{j-1}+1\right)}^{N} & b_{n_{i}\left(m_{j-1}+2\right)}^{N} & \cdots & b_{n_{i} m_{j}}^{N}
\end{array}\right] \\
& \in \mathbf{R}^{\left(n_{j}-n_{i}\right) \times\left(m_{j}-m_{i}\right)} .
\end{aligned}
$$

Moreover, let us denote $\widetilde{\mathbf{B}}_{l}^{N}$ by

$$
\widetilde{\mathbf{B}}_{l}^{N}=\left[\begin{array}{cccc}
\widetilde{\mathbf{B}}_{11}^{N} & \widetilde{\mathbf{B}}_{12}^{N} & \cdots & \widetilde{\mathbf{B}}_{1 l}^{N} \\
\mathbf{0} & \widetilde{\mathbf{B}}_{22}^{N} & \cdots & \widetilde{\mathbf{B}}_{2 l}^{N} \\
\vdots & \vdots & \ddots & \vdots \\
\mathbf{0} & \mathbf{0} & \cdots & \widetilde{\mathbf{B}}_{l l}^{N}
\end{array}\right] \in \mathbf{R}^{n_{l} \times m_{l}}
$$

By (17), the effects of normal actuators can be explicitly described. Then the reconfiguration criterion can be stated as follows. 
Reconfiguration Criterion. The system that has $k$-faulty actuators is reconfigurable, if a matrix $\widetilde{\mathbf{B}}_{l}^{N} \in \mathbf{R}^{n_{l} \times m_{l}}$ satisfies the following:

(i) $\operatorname{rank}\left(\widetilde{\mathbf{B}}_{l}^{N}\right) \geq r$;

(ii) $\widetilde{\mathbf{B}}_{l}^{N}$ is full row rank; that is, $\operatorname{rank}\left(\widetilde{\mathbf{B}}_{l}^{N}\right)=n_{l}$.

From conditions (i) and (ii), there exists at least one solution $\mathbf{u}_{2}^{R}$ that satisfies the following:

$$
\widetilde{\mathbf{B}}_{l}^{N} \mathbf{u}_{2}^{R}=\left[\begin{array}{c}
-\mathbf{B}_{11}^{F} \mathbf{e}^{u} \\
\mathbf{0}_{\left(n_{l}-r\right)}
\end{array}\right]=\left[\begin{array}{c}
-\mathbf{e}^{v} \\
\mathbf{0}_{\left(n_{l}-r\right)}
\end{array}\right] .
$$

Equation (18) means that the effects of faulty actuators can be compensated by $n_{l}$-normal actuators. This leads to the fact that the required number of normal actuators for tolerating faults is $n_{l}$. Hence, the minimum number of redundant actuators can be stated as follows.

Minimum Number of Redundant Actuators. If the system is reconfigurable for $k$-faulty actuators, that is, for $l \leq t \leq m$, there exists a matrix $\widetilde{\mathbf{B}}_{t}^{N}$ that satisfies the reconfiguration criterion, then the minimum number of redundant actuators is $n_{l}$.

It is worth noting that the faulty system that satisfies the reconfiguration criterion can compensate the effects of faults by redistributing normal actuators. However, if a system cannot take sufficient normal actuators, then reconfiguration cannot be achieved; consequently, the effects of faulty actuators may cause catastrophic accident. In this case, uncontrolled forces generated by faulty actuators stress the system structures. Hence, the primary goal of faulttolerant control is to achieve stabilization of the system by degrading the performance of normal actuators. Figure 2 describes the fault-tolerant control strategy. In Figure 2, fault-tolerance can be achieved by two ways: performance maintenance and stabilization (graceful degradation). If the system satisfies the proposed reconfiguration criterion, then the effects of the faulty actuators can be compensated; that is, the performance of the faulty system can be maintained. In contrast, if the system cannot achieve perfect compensation due to lack of redundant actuators, that is, the reconfiguration criterion cannot be satisfied, then the fault-tolerant mechanism tries to stabilize the system by reducing the required performance in order to avoid additional types of damage such as structural breaks. Examples of fault-tolerant control strategy for these two types are introduced in the following section.

\section{Examples}

In this section, two systems are introduced to show how to work the proposed reconfiguration criterion. The first example is a simple crane system that has not any sufficiently redundant normal actuators for tolerating fault. The other example is an aircraft that adopts various control surfaces (actuators).

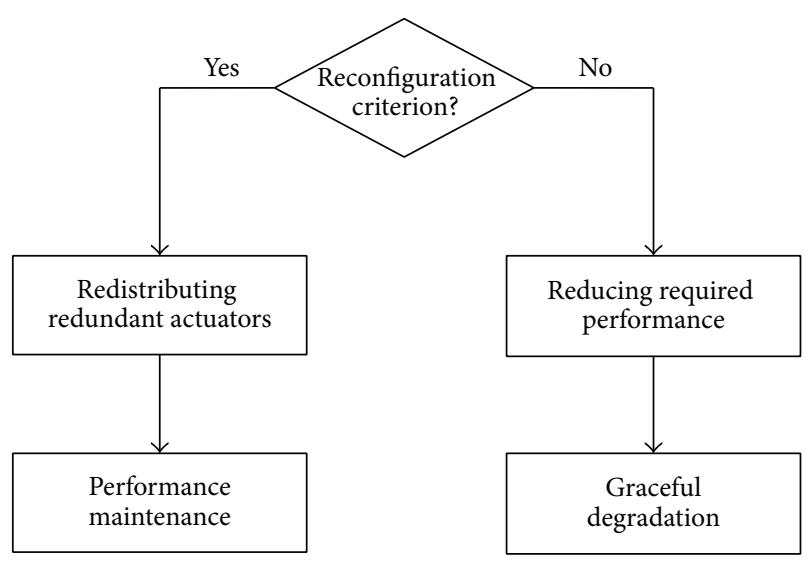

FIGURE 2: Fault-tolerant control strategy.

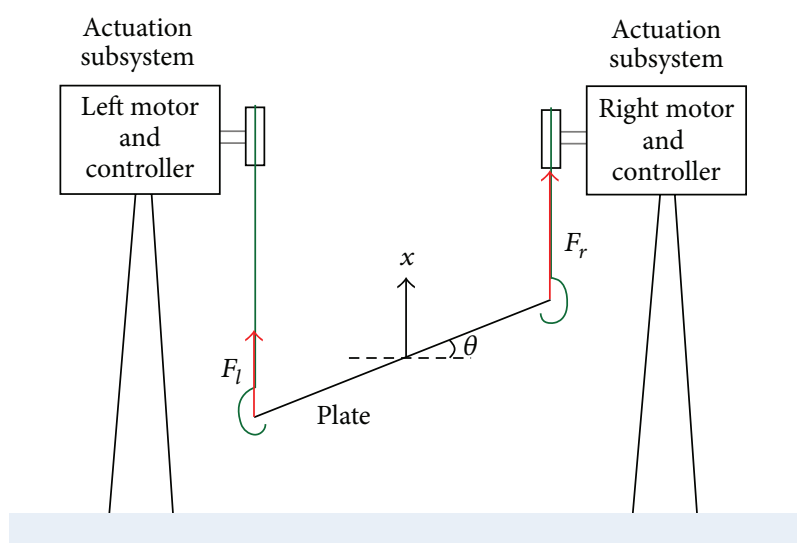

FIgURE 3: Description of the crane model.

4.1. Crane System. A simple crane system considered in this subsection is depicted in Figure 3. In this crane, center of the horizontal plate is moved up and down by two motors:

$$
F_{l}+F_{r}-m g=m a,
$$

where $m$ is the plate mass and $g$ is the gravitational constant. And $F_{l}$ and $F_{r}$ are forces generated by the left and right motors, respectively. To control the plate position (height), a dynamic inversion control law applies to both motors.

In this simulation, it is assumed that a $30 \%$ of LoE fault occurs on the left motor; that is, the force generated by the left motor is reduced by almost $30 \%$. Then the plate is operated by asymmetric forces; consequently, the plate cannot be stabilized. Since forces are generated asymmetrically, the following moment equation is applied to the plate:

$$
F_{r} l \cos \theta-F_{l} l \cos \theta=J \ddot{\theta},
$$

where $J$ is the rotational inertia of the plate and $\theta$ is the angle between the horizontal line and the plate. And $l$ indicates the distance from the center of the plate to the position that force $F_{l}$ or $F_{r}$ activates.

So fault occurring on the left motor affects two equations, (19) and (20); that is, $r=2$. However, the number of 
the remaining normal motor is one (right motor). Hence, the reconfiguration criterion cannot be satisfied.

In Figure 4, responses of plate position and angle of the faulty crane are described. Although fault on the left motor (actuator) degrades its performance, the controlled plate position can maintain the referenced position. However, since the controller generates more forces to satisfy the referenced plate position, the plate angle is oscillated (Figure 4(b)). As a result, the faulty crane cannot be stable.

From the reconfiguration criterion result, the crane system cannot achieve sufficient number of normal motors that can accommodate the effects of fault. In this case, the primary goal of fault-tolerant control is to stabilize the faulty crane; that is, fault-tolerant mechanism tries to reduce the required performance of the crane. Figure 5 shows the simulation results of the performance maintenance crane case by applying the reconfiguration input introduced in (14). In Figure 5(a), the control performance can be maintained by generating additional force to the normal (right) motor. However, the plate is extremely oscillated due to asymmetric additional force acting on the normal motor (Figure 5(b)). Moreover, this additional force may cause structural damage, if the crane is oscillated continuously. In contrast, in Figure 6(b), oscillation of the plate can be prevented by degrading the performance of the normal motor. Although control performance cannot be achieved as shown in Figure 6(a), the faulty crane can avoid additional damage.

4.2. Aircraft System. In this paper, a tailless jet fighter developed under the innovative control effectors (ICE) program is considered [4, 12]. The ICE adopts elevons, pitch flap, and all-moving tips as control surfaces. Since the proposed reconfiguration criterion represents the relationship between faulty states and normal actuators, the input distribution matrix of the ICE is considered in this simulation. In [12], the input distribution matrix linearized at Mach 0.4 and $15,000 \mathrm{ft}$ altitude yields

$$
\begin{aligned}
& {\left[\begin{array}{l}
v_{p} \\
v_{q} \\
v_{r}
\end{array}\right]=\left[\begin{array}{rrrrr}
3.7830 & -3.7830 & 0.0000 & 1.8255 & -1.8255 \\
-2.5114 & -2.5114 & -1.9042 & -0.9494 & -0.9494 \\
-0.0453 & -0.0453 & 0.0000 & -0.2081 & 0.2081
\end{array}\right]} \\
& \times\left[\begin{array}{c}
\delta_{\mathrm{le}} \\
\delta_{\mathrm{re}} \\
\delta_{\mathrm{pf}} \\
\delta_{\text {lamt }} \\
\delta_{\text {ramt }}
\end{array}\right],
\end{aligned}
$$

where $v_{p}, v_{q}$, and $v_{r}$ denote the virtual inputs for roll rate, pitch rate, and yaw rate, respectively. And control inputs, $\delta_{\mathrm{le}}$, $\delta_{\text {re }}, \delta_{\text {pf }}, \delta_{\text {lamt }}$, and $\delta_{\text {ramt }}$, are deflections of left elevon, right elevon, pitch flap, left all-moving tip, and right all-moving tip, correspondingly.

In this simulation, the following fault scenario is considered: a $30 \%$ of LoE fault on the left elevon at $4 \mathrm{sec}$ and float fault on the right all-moving tip at $8 \mathrm{sec}$. Since one fault occurs on the left elevon during 4-8 sec, the input distribution matrix $\left[\begin{array}{ll}\mathbf{B}_{1}^{F} & \mathbf{B}_{2}^{N}\end{array}\right]$ can be represented as follows:

$$
\begin{aligned}
& \mathbf{B}_{1}^{F}=\left[\begin{array}{r}
-2.5114 \\
3.7830 \\
0.0000
\end{array}\right], \\
& \mathbf{B}_{2}^{N}=\left[\begin{array}{rrrr}
-1.9042 & -2.5114 & -0.9494 & -0.9494 \\
0.0000 & -3.7830 & 1.8255 & -1.8255 \\
0.0000 & 0.0000 & -0.2081 & 0.2081
\end{array}\right] .
\end{aligned}
$$

Since the effects of elevons in $v_{r}$ are much less than other control surfaces in (22), it is reasonable that -0.0453 is set to be 0 . The number of affected virtual inputs is 2 ; that is, $r=2$. And $\widetilde{\mathbf{B}}_{l}^{N}(l=1,2,3)$ can be represented as

$$
\begin{gathered}
\widetilde{\mathbf{B}}_{1}^{N}=[-1.9042], \quad \widetilde{\mathbf{B}}_{2}^{N}=\left[\begin{array}{rrr}
-1.9042 & -2.5114 \\
0.0000 & -3.7830
\end{array}\right], \\
\widetilde{\mathbf{B}}_{3}^{N}=\left[\begin{array}{rrrr}
-1.9042 & -2.5114 & -0.9494 & -0.9494 \\
0.0000 & -3.7830 & 1.8255 & -1.8255 \\
0.0000 & 0.0000 & -0.2081 & 0.2081
\end{array}\right] .
\end{gathered}
$$

To examine the possibility of reconfiguration, select $\widetilde{\mathbf{B}}_{2}^{N}$; then, the following are satisfied:

(i) $\operatorname{rank}\left(\widetilde{\mathbf{B}}_{2}^{N}\right)=2=r$;

(ii) $\widetilde{\mathbf{B}}_{2}^{N}$ is full row rank.

So the reconfiguration criterion is satisfied. Hence, the effects of faulty left elevon during $4-8 \mathrm{sec}$ can be compensated. Moreover, the minimum required number of normal actuators is 2. Actually, the reconfiguration criterion can also be satisfied, if $\widetilde{\mathbf{B}}_{3}^{N}$ is selected.

Similarly, for two-faulty-actuator case after $8 \mathrm{sec}$, the input distribution matrix $\left[\begin{array}{ll}\mathbf{B}_{1}^{F} & \mathbf{B}_{2}^{N}\end{array}\right]$ yields

$$
\begin{aligned}
& \mathbf{B}_{1}^{F}=\left[\begin{array}{rr}
-2.5114 & -0.9494 \\
3.7830 & -1.8255 \\
0.0000 & 0.2081
\end{array}\right], \\
& \mathbf{B}_{2}^{N}=\left[\begin{array}{rrr}
-1.9042 & -2.5114 & -0.9494 \\
0.0000 & -3.7830 & 1.8255 \\
0.0000 & 0.0000 & -0.2081
\end{array}\right] .
\end{aligned}
$$

Then $r=3$. And for $l \in[1,3], \widetilde{\mathbf{B}}_{l}^{N}$ can be represented as

$$
\begin{gathered}
\widetilde{\mathbf{B}}_{1}^{N}=[-1.9042], \quad \widetilde{\mathbf{B}}_{2}^{N}=\left[\begin{array}{rrr}
-1.9042 & -2.5114 \\
0.0000 & -3.7830
\end{array}\right], \\
\widetilde{\mathbf{B}}_{3}^{N}=\left[\begin{array}{rrr}
-1.9042 & -2.5114 & -0.9494 \\
0.0000 & -3.7830 & 1.8255 \\
0.0000 & 0.0000 & -0.2081
\end{array}\right] .
\end{gathered}
$$

Choose $\widetilde{\mathbf{B}}_{3}^{N}$; then, the following are satisfied:

(i) $\operatorname{rank}\left(\widetilde{\mathbf{B}}_{3}^{N}\right)=3=r$;

(ii) $\widetilde{\mathbf{B}}_{3}^{N}$ is full row rank. 


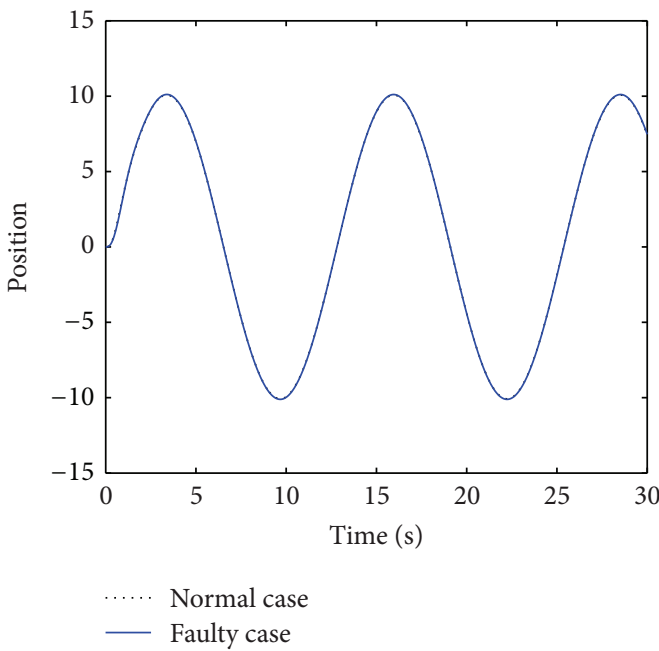

(a) Position response

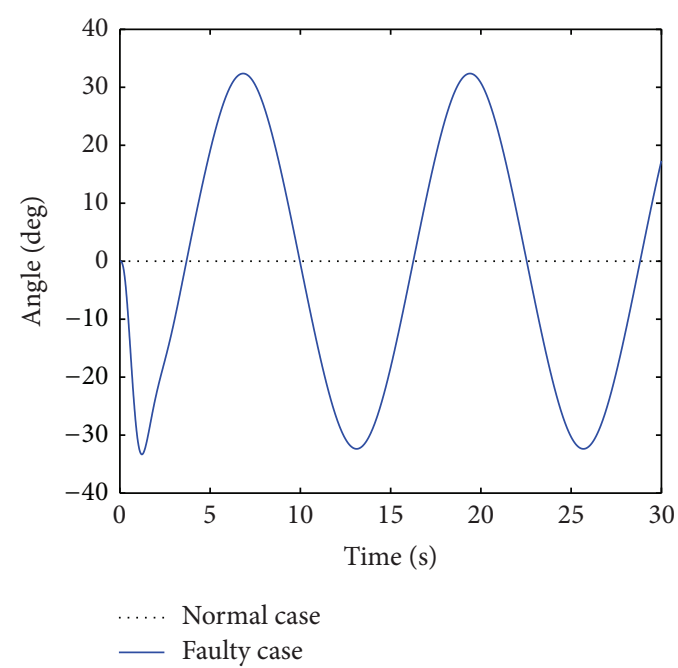

(b) Plate angle response

FIGURE 4: Results of faulty crane case.

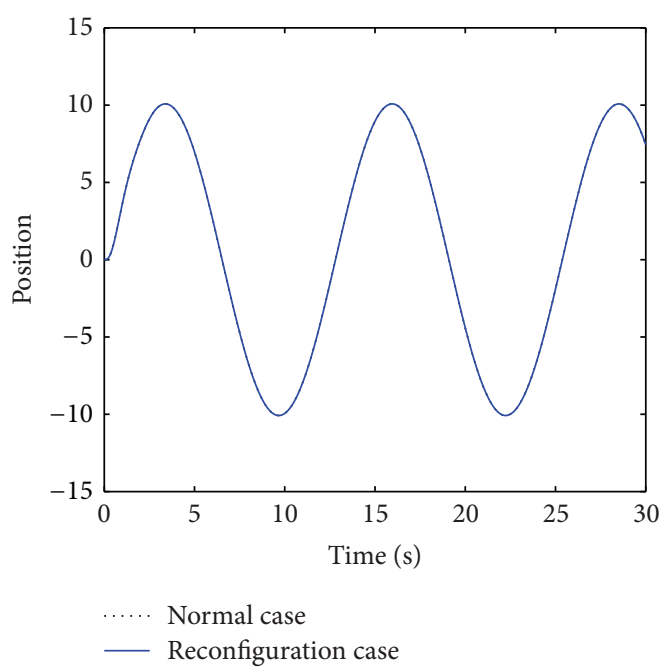

(a) Position response

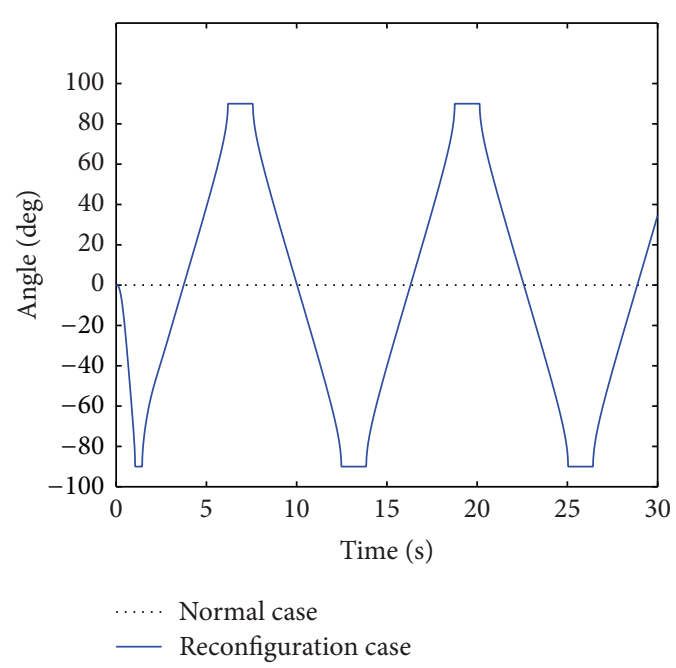

(b) Plate angle response

FIGURE 5: Results of performance maintained crane case.

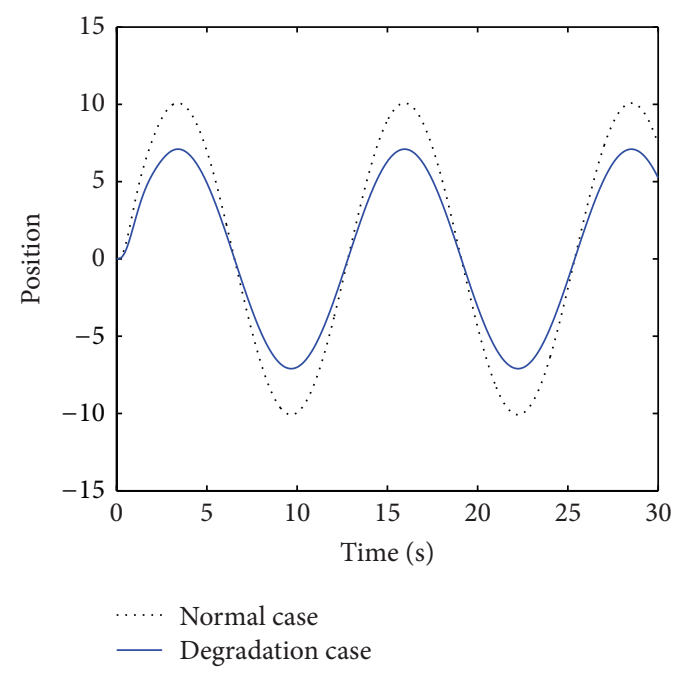

(a) Position response

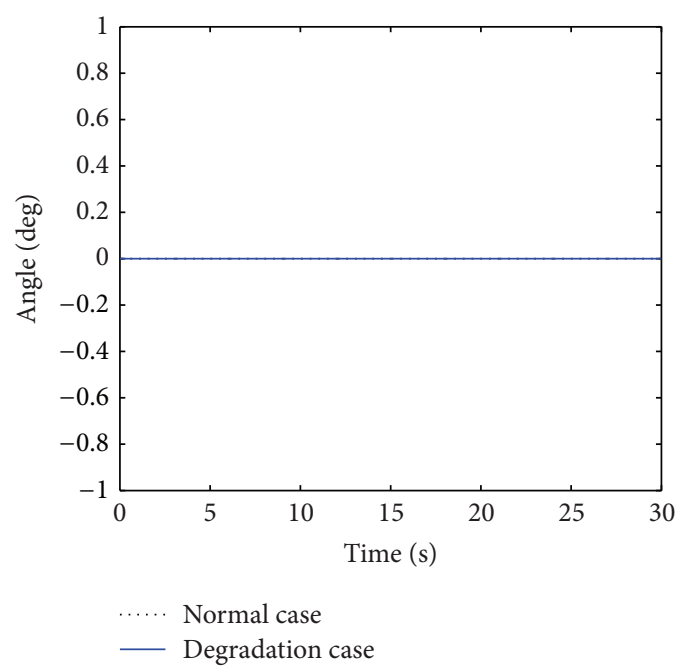

(b) Plate angle response

FIGURE 6: Results of performance degraded crane case. 


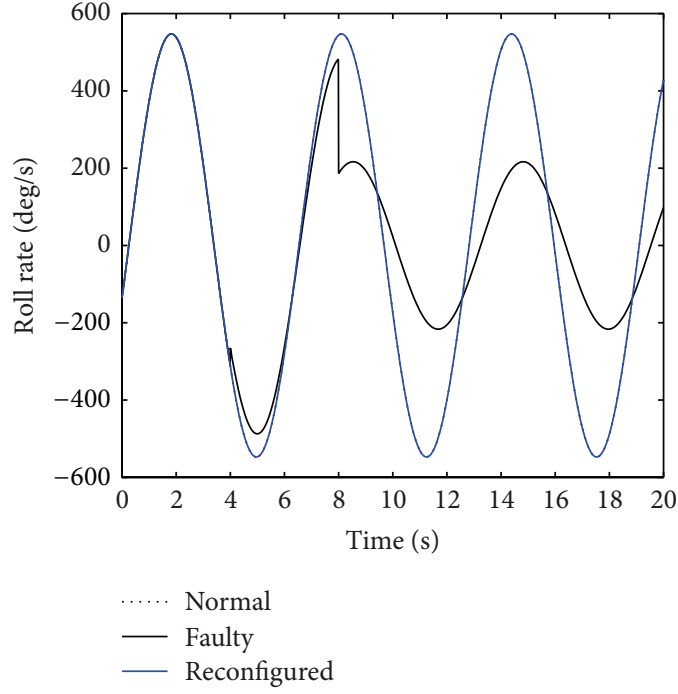

(a) Virtual input response of roll rate

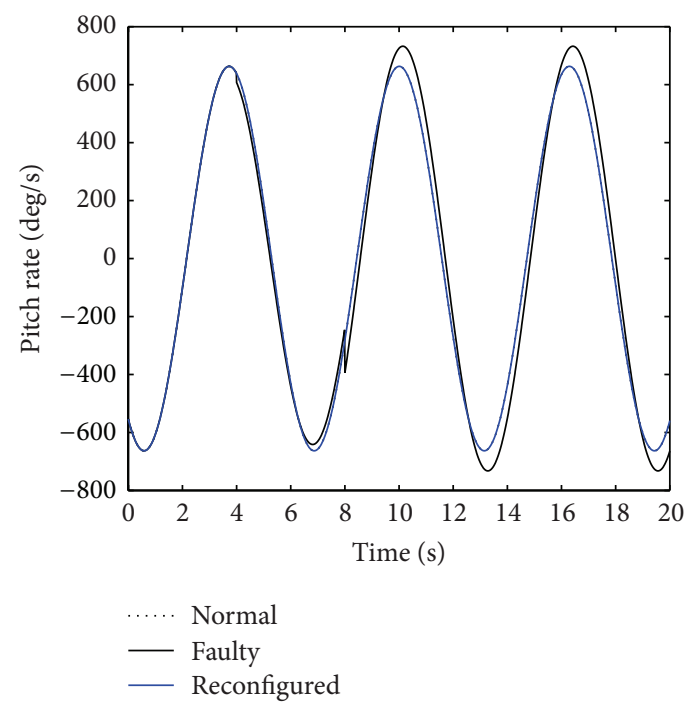

(b) Virtual input response of pitch rate

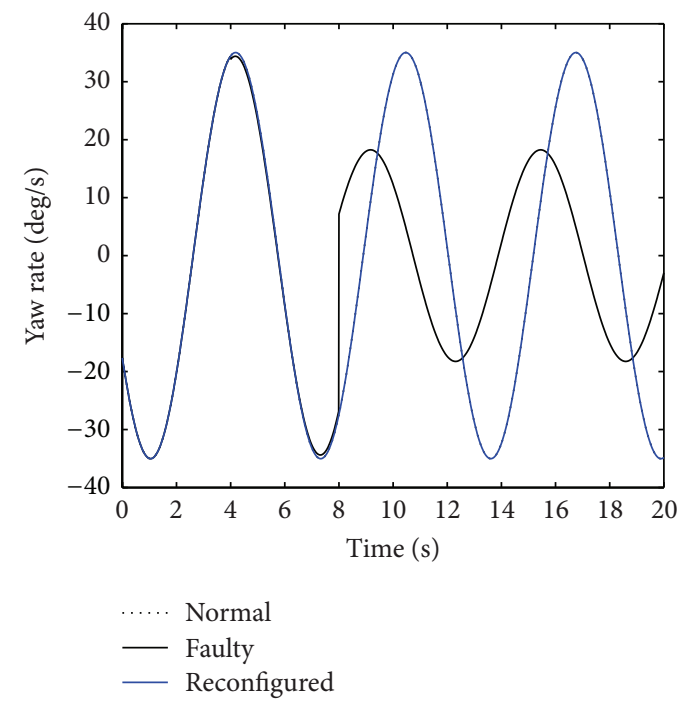

(c) Virtual input response of yaw rate

FIGURE 7: Virtual input response with/without reconfiguration.

So the reconfiguration criterion is satisfied. Therefore, the effects of faulty left elevon and right all-moving tip after $8 \mathrm{sec}$ can be accommodated. Moreover, it is easy to check that the minimum required number of normal actuators is 3. Hence, faults occurring in the ICE can be compensated by the redundant normal actuators.

Figure 7 shows the simulation results. In these figures, relationships between the virtual inputs and actual control surfaces are considered. Due to fault on the left elevon at $4 \mathrm{sec}$, the performances of virtual inputs are degraded. Moreover, the performances of virtual inputs in roll and yaw dynamics are significantly reduced after injecting float fault on the right all-moving tip at $8 \mathrm{sec}$. However, by redistributing the normal actuators such as right elevon, pitch flap, left all-moving tip, and right all-moving tip (before $8 \mathrm{sec}$ ), the effects of injected faults can be compensated.
The following figures show the results of roll motion of the faulty ICE. In this simulation, the aircraft maneuvers turn reversal which can be achieved by rolling the aircraft to $60 \mathrm{deg}$ to the left and then to $60 \mathrm{deg}$ to the right. As shown in Figure 8(b), the roll angle diverges due to injected fault on the left elevon at $4 \mathrm{sec}$. However, by redistributing the normal actuators, the effects of faulty actuator can be accommodated; consequently, the aircraft can maintain the performance as shown in Figure 8(c).

\section{Concluding Remarks}

This paper has proposed the reconfiguration criterion that presents the possibility of fault-tolerance. The proposed criterion explicitly describes the relationship between the faulty states and normal actuators. This relationship determines 


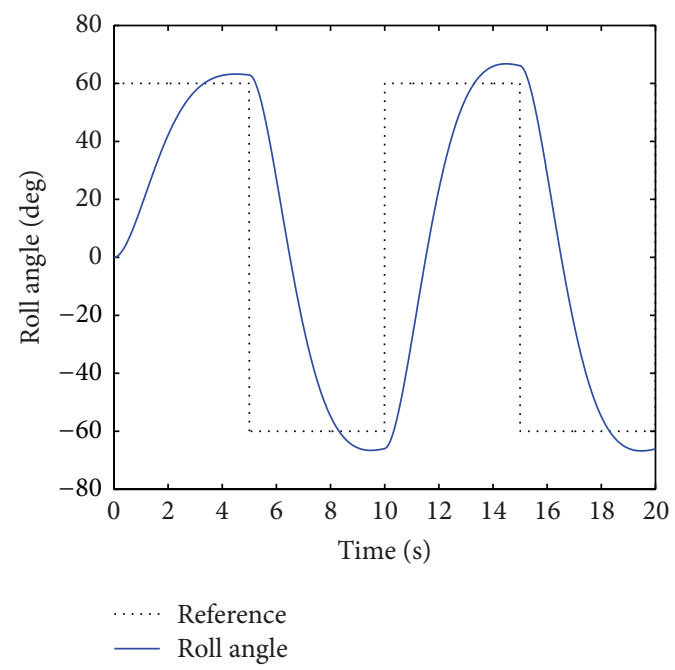

(a) Normal case

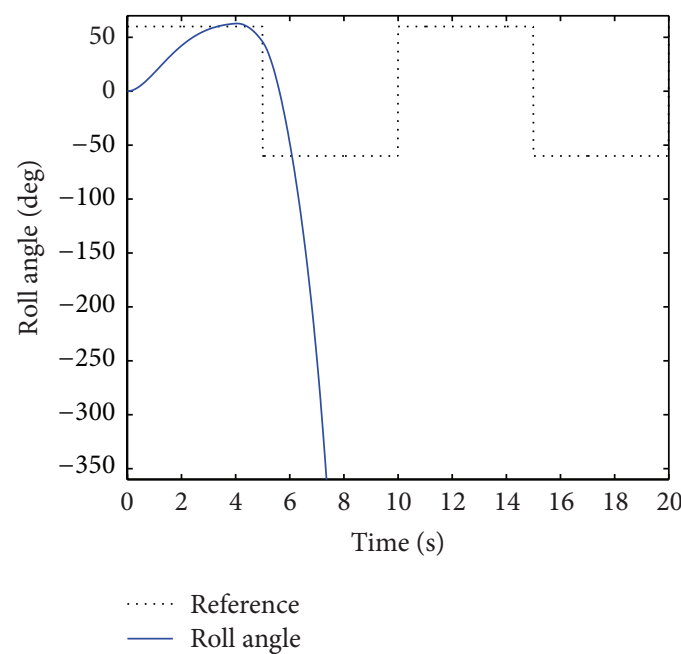

(b) Without reconfiguration

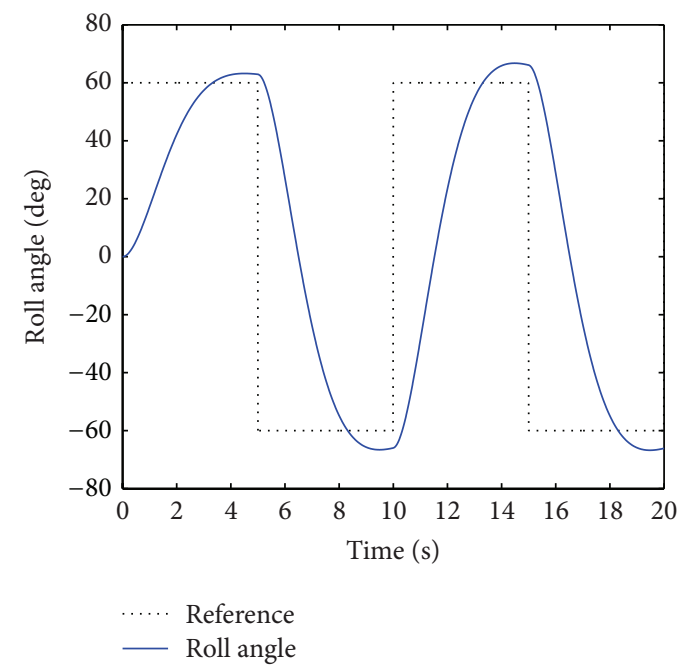

(c) With reconfiguration

FIgURE 8: Turn reversal maneuver response.

whether the effects of faulty actuators can be compensated by normal actuators or not. Moreover, the relationship also provides the required number of normal actuators for faulttolerance. Hence, the proposed criterion can be used to determine the goals of fault-tolerance: performance maintenance or graceful degradation.

However, the reconfiguration criterion proposed in this paper is analyzed for a linearized system. For future work, the proposed criterion will be extended to a nonlinear system. Moreover, actuator limitation called saturation will be considered to adopt the proposed criterion in physical systems.

\section{Conflict of Interests}

The authors declare that there is no conflict of interests regarding the publication of this paper.

\section{Acknowledgment}

This research was supported by the MSIP (Ministry of Science, ICT \& Future Planning), Korea, under the C-ITRC (Convergence Information Technology Research Center) support program (NIPA-2014-H0401-14-1004) supervised by the NIPA (National IT Industry Promotion Agency).

\section{References}

[1] Z. Gao and P. J. Antsaklis, "Stability of the pseudo-inverse method for reconfigurable control systems," International Journal of Control, vol. 53, no. 3, pp. 717-729, 1991.

[2] K. J. Åström and B. Wittenmark, Adaptive Control, AddisonWesley, 2nd edition, 1995.

[3] H. Alwi and C. Edwards, "Fault tolerant control using sliding modes with on-line control allocation," Automatica, vol. 44, no. 7, pp. 1859-1866, 2008. 
[4] Y. Shtessel, J. Buffington, and S. Banda, "Tailless aircraft flight control using multiple time scale reconfigurable sliding modes," IEEE Transactions on Control Systems Technology, vol. 10, no. 2, pp. 288-296, 2002.

[5] I. Yang, Y.-J. Kim, and D. Lee, "Actuator failure diagnosis and accommodation using sliding mode control for submersible vehicle," Journal of Institute of Control, Robotics and Systems, vol. 16, no. 7, pp. 661-667, 2010.

[6] J. D. Bošković and R. K. Mehra, "Multiple model-based reconfigurable flight control system design," in Proceedings of the 37th IEEE Conference on Decision and Control, pp. 4503-4508, December 1998.

[7] D. Enns, "Control allocation approaches," in AIAA Guidance, Navigation and Control Conference and Exhibit, pp. 98-108, 1998.

[8] J. Buffington, P. Chandler, and M. Pachter, "On-line system identification for aircraft with distributed control effectors," International Journal of Robust and Nonlinear Control, vol. 9, no. 14, pp. 1033-1049, 1999.

[9] I. Yang, D. Kim, and D. Lee, "Fault-tolerant control strategy based on control allocation using smart actuators," in Proceedings of the 1st Conference on Control and Fault-Tolerant Systems (SysTol '10), pp. 377-381, Nice, France, October 2010.

[10] I. Yang, D. Kim, and D. Lee, "Fault-tolerant networked control systems using control allocation for failures in multiple control surfaces," Journal of Institute of Control, Robotics and Systems, vol. 17, no. 11, pp. 1067-1073, 2011.

[11] J. D. Bošković and R. K. Mehra, "Failure detection, identification and reconfiguration in flight control," in Fault Diagnosis and Fault Tolerance for Mechatronic Systems: Recent Advances, vol. 1 of Springer Tracts in Advanced Robotics, pp. 129-167, Springer, Berlin, Germany, 2003.

[12] J. Buffington, "Tailless aircraft control allocation," in Proceedings of the AIAA Guidance, Navigation and Control Conference, AIAA 97-3605, pp. 737-747, New Orleans, La, USA, 1997. 


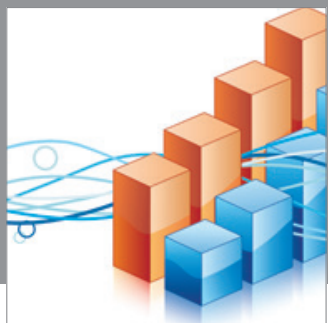

Advances in

Operations Research

mansans

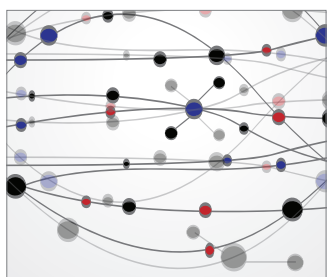

The Scientific World Journal
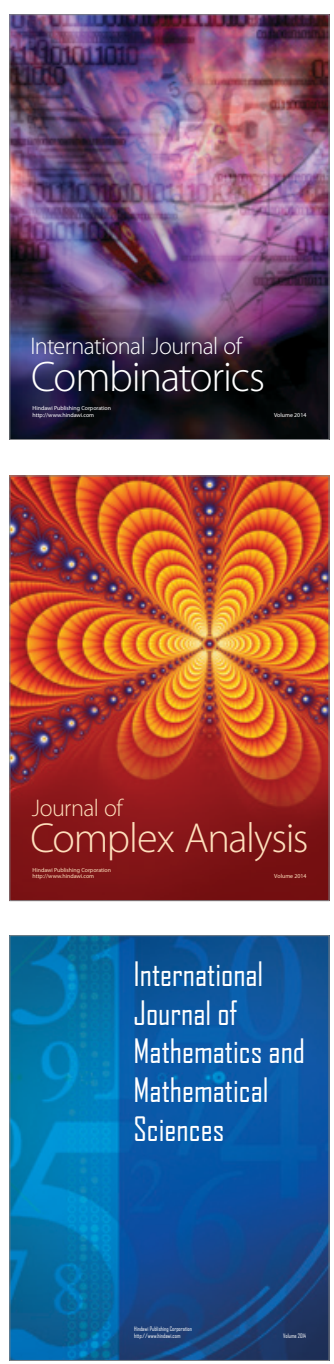
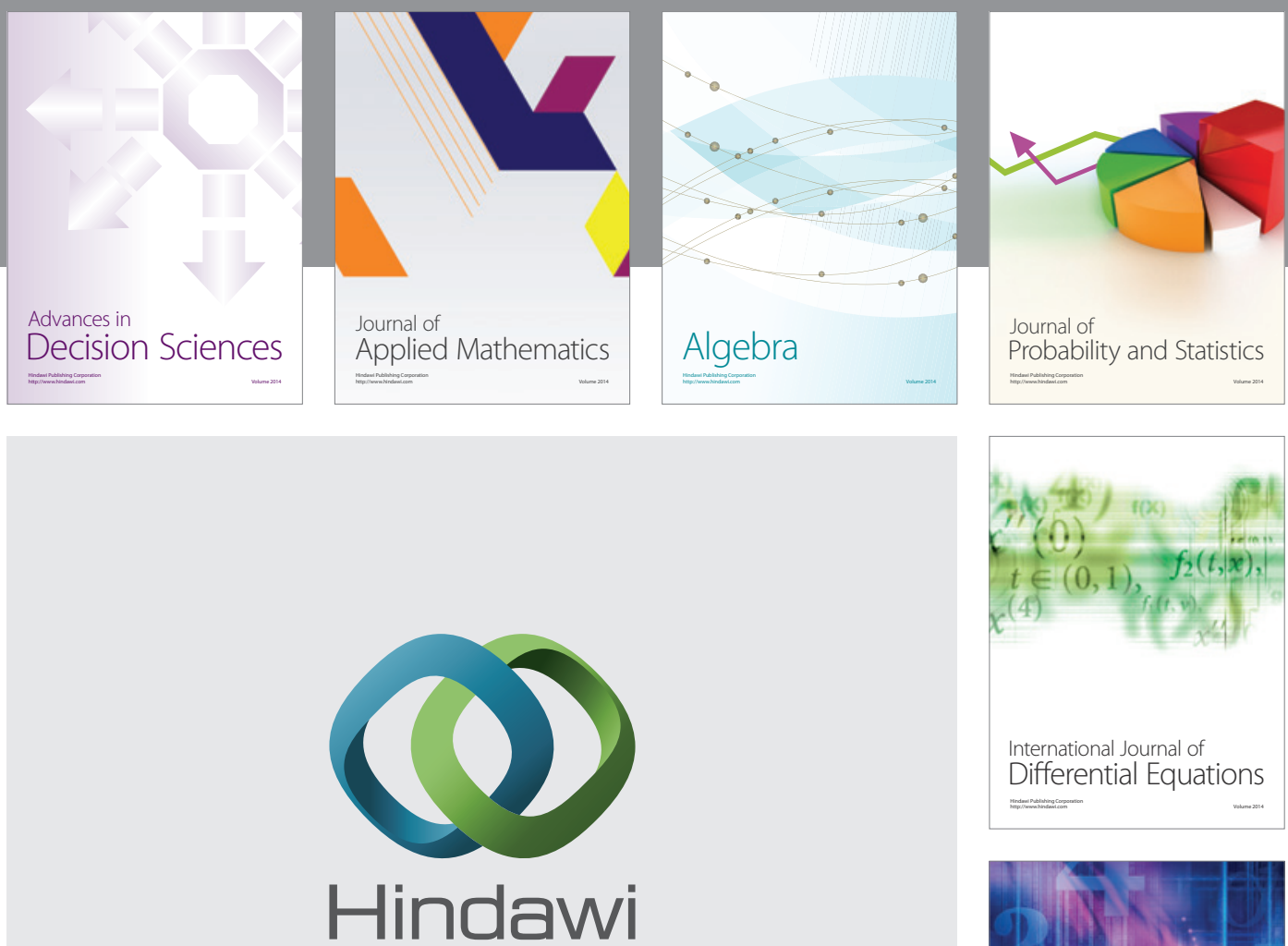

Submit your manuscripts at http://www.hindawi.com
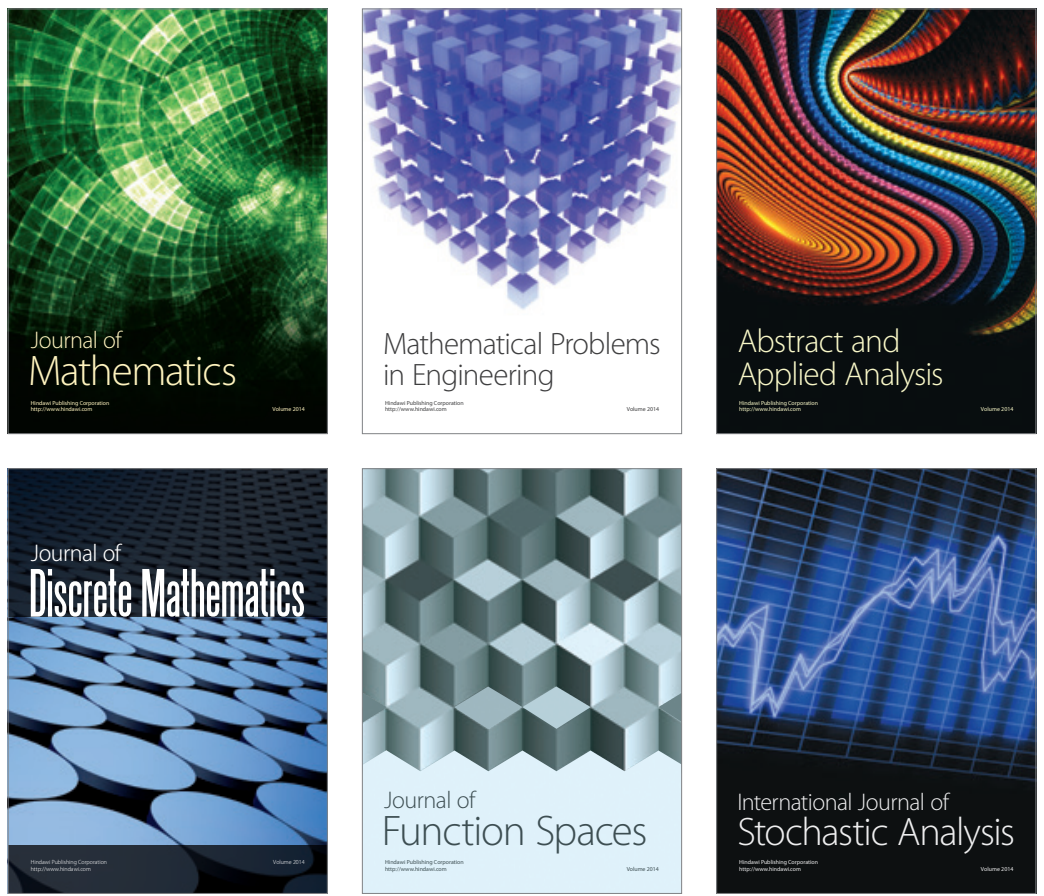

Journal of

Function Spaces

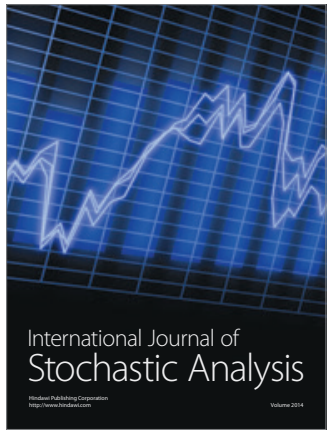

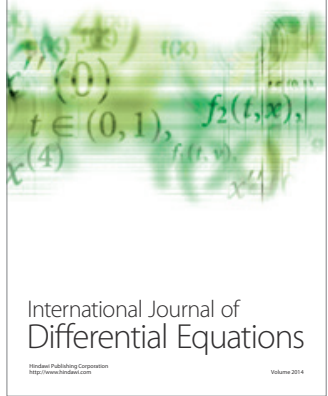
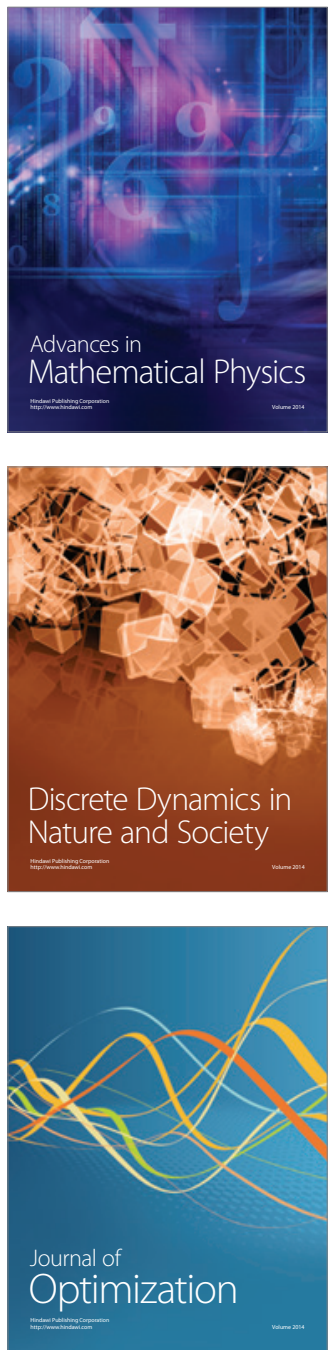